\title{
Research on Precharge Control Strategy of Modular Multilevel Inverter
}

\author{
Chuanliang Fang ${ }^{1}$, Guochu Chen ${ }^{1, *}$ \\ ${ }^{1}$ Electrical Engineering, Shanghai DianJi University, Shanghai, China
}

\begin{abstract}
This paper mainly introduces the Modular Multilevel Converter (MMC) topology structure, simple analysis of the working principle of MMC, deduced the mathematical model of MMC. The precharging control strategy of MMC sub-module (SM) is studied, and the charging process is divided into uncontrollable charging stage and controllable charging stage. For the uncontrollable charging stage, an improved DC side pre-charging method is proposed, and a five-level simulation model of voltage-type MMC is established in the Matlab/Simulink simulation environment, and the simulation results of the system are comprehensively analyzed.
\end{abstract}

\section{Introduction}

Modular Multilevel Converter (MMC), as an important part of new flexible DC power transmission technology, has the characteristics of small switch loss, low output voltage harmonic content, high degree of modularization and easy to expand, and has been widely used in the field of DC power transmission ${ }^{[1]}$. Domestic and foreign experts and scholars mainly focus on circulation suppression, voltage equalization control of submodules(SM) and redundancy control, etc. However, researches on these issues have assumed that MMC capacitance and rated voltage have been established, and relatively few researches on pre-charging of MMC submodules have been conducted. Current studies mainly focus on the pre-charging of MMC through the $\mathrm{AC}$ side, while there are relatively few studies on the pre-charging of the DC side. Literature [1] proposed a method to charge submodules by controlling the charging current, which needs to consider the charging current under different circuit states, and the method is relatively complex. Literature [2] proposed a DC side active charging strategy by gradually decreasing the number of input sub-modules. This method started from the controllable charging stage and did not take into account the influence of current limiting resistance on charging effect.

In view of the shortcomings of the current system pre-charging research process, based on the existing DC side pre-charging methods, this paper proposes a method of controlling the current limiting resistor in the uncontrollable charging stage to precharge the submodules, and the simulation results verify the feasibility of the method presented in this paper.

\section{Materials and Methods}

\subsection{MMC basic theory}

The main circuit of three-phase MMC inverter is shown in Figure 1. The MMC system has a total of 6 bridge arms, each bridge arm contains 1 inductor $\mathrm{L}$ and $\mathrm{N}$ cascade sub-modules (SM), and the upper and lower two bridge arms of each phase are a phase unit of MMC. The inductance of the bridge arm can suppress the shortcircuit impulse current when the DC bus is short-circuit fault.

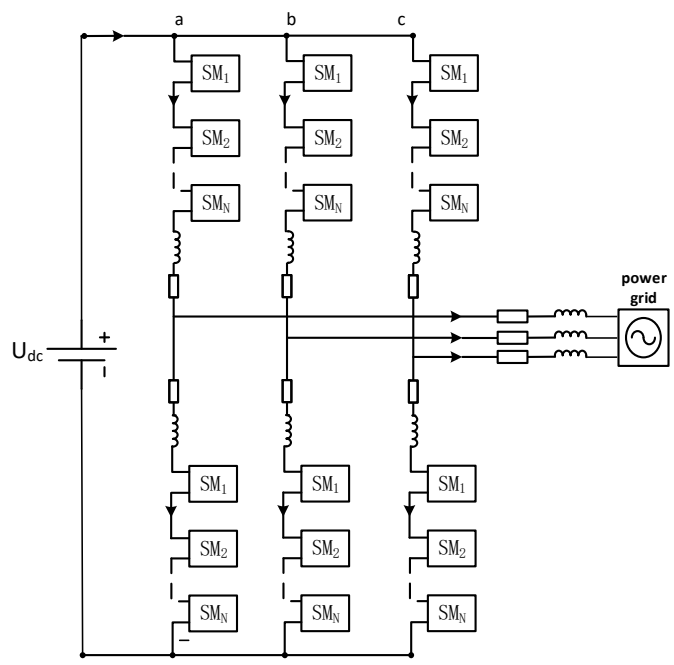

Fig. 1. MMC main circuit topology structure

Due to the low loss and low cost of the Half Bridge Sub-Module (HBSM), most of the current MMC submodule topologies adopt HBSM. Its topology is shown in Figure 2. 


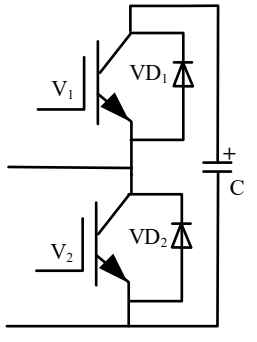

Fig. 2. Sub-module topology

HBSM is composed of two $\operatorname{IGBT}\left(\mathrm{V}_{1}, \mathrm{~V}_{2}\right)$ switches in series, two inverse parallel diodes $\left(\mathrm{VD}_{1}, \mathrm{VD}_{2}\right)$ and a capacitor(C). The parameters of submodule and inductance on each arm of the half-bridge structure are exactly the same. The output voltage of MMC is determined by the working state of each unit sub-module. By controlling the switching on and off of each unit submodule, each sub-module can work in three different states: input, cut off or lock, and the corresponding submodule capacitance can be switched in three states: charging, discharging and bypass. Thus, port voltage $\mathrm{U}_{\mathrm{SM}}$ is switched between $\mathrm{UC}$ and $0^{[3-7]}$. The working state of the half bridge sub-module is shown in Table 1.

Table 1. MMC sub-module working mode

\begin{tabular}{|c|c|c|c|}
\hline Mode & $\begin{array}{c}\text { Switch } \\
\text { tube state }\end{array}$ & Current path & $\begin{array}{c}\text { SM port } \\
\text { voltage }\end{array}$ \\
\hline Charging & $\begin{array}{c}\mathrm{V}_{1} \text { on } \\
\mathrm{V}_{2} \text { off }\end{array}$ & $\mathrm{VD}_{1}$ & $\mathrm{U}_{\mathrm{C}}$ \\
\hline Discharging & $\begin{array}{l}\mathrm{V}_{1} \text { on } \\
\mathrm{V}_{2} \text { off }\end{array}$ & $\mathrm{V}_{1}$ & $\mathrm{U}_{\mathrm{C}}$ \\
\hline Resection & $\begin{array}{l}\mathrm{V}_{1} \text { off } \\
\mathrm{V}_{2} \text { on }\end{array}$ & $\mathrm{V}_{2}$ or $\mathrm{VD}_{2}$ & 0 \\
\hline Block & $\begin{array}{l}\mathrm{V}_{1} \text { off } \\
\mathrm{V}_{2} \text { off }\end{array}$ & $\mathrm{VD}_{1}$ or $\mathrm{VD}_{2}$ & 0 or $\mathrm{U}_{\mathrm{C}}$ \\
\hline
\end{tabular}

\subsection{The mathematical model of MMC}

Phase $\mathrm{A}$ is taken as an example to analyze, establishing a mathematical model for phase $\mathrm{A}$, and its equivalent circuit diagram is shown in Figure 3.

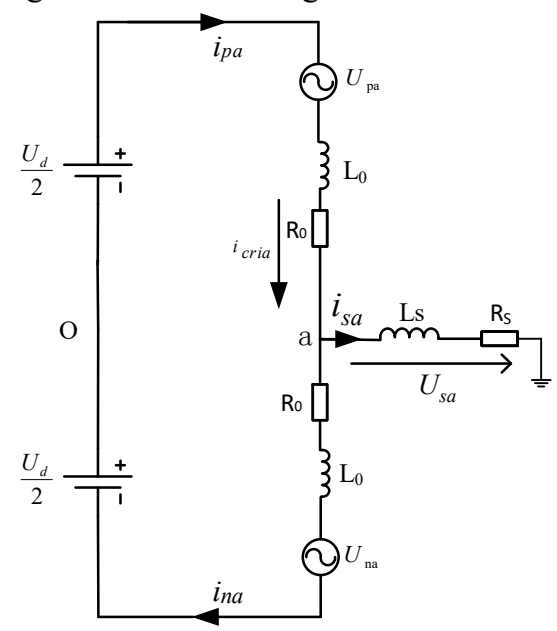

Fig. 3. MMC inverter equal value circuit of Phase A
In figure $3, \mathrm{U}_{\mathrm{d}}$ represents the $\mathrm{DC}$ side voltage, $\mathrm{U}_{\mathrm{pa}}$ and $U_{n a}$ represent the total equivalent output voltage of the upper and lower bridge arms $\mathrm{N}$ sub-modules, $\mathrm{i}_{\mathrm{pa}}$ and $i_{\text {na }}$ are the currents of the upper and lower bridge arms, each bridge arm is connected in series with a reactor $\mathrm{L}_{0}$ and the equivalent resistance of the bridge arm $R_{0}$. $i_{\text {cria }}$ is the internal circulating current flowing through the upper and lower bridge arms of phase $A . U_{\mathrm{sa}}$ and $\mathrm{i}_{\mathrm{sa}}$ are the voltage and load current output by the $\mathrm{AC}$ side of phase A. $R_{s}$ and $L_{s}$ are the resistive and inductive load output on the AC side.

To ensure that the DC bus voltage remains unchanged, the number of SM for each phase unit is $\mathrm{N}$ :

$$
N=N_{p a}+N_{n a}
$$

Among them, $\mathrm{N}_{\mathrm{pa}}$ is the number of sub-modules for the upper bridge arm, and $\mathrm{N}_{\mathrm{na}}$ is the number of submodules for the lower bridge arm. The voltage across the capacitor of each sub-module is:

$$
U_{c}=\frac{U_{d}}{N}
$$

Write the KCL equation to point a, the relationship between the output current $i_{\text {sa }}$ and the bridge arm currents can be obtained:

$$
i_{s a}=i_{p a}-i_{n a}
$$

Consider the interphase circulating current $i_{\text {cria }}$, the relationship between the load current and circulating current is as follows:

$$
\begin{aligned}
& i_{n a}=i_{c r i a}-\frac{i_{s a}}{2} \\
& i_{n a}=i_{c r i a}+\frac{i_{s a}}{2}
\end{aligned}
$$

From the above formula, the internal circulation expression of A-phase bridge arm can be obtained:

$$
i_{\text {cria }}=\frac{i_{p a}+i_{n a}}{2}
$$

Ignore the mutual inductance between the upper and lower arm reactors, writing KVL of Figure 3 to deduce the output voltage of phase A:

$$
U_{s a}=\frac{U_{n a}-U_{p a}}{2}-\frac{L_{0}}{2} \frac{d i_{s a}}{d t}-R_{0} i_{s a}
$$

It can be seen from equation (6) that there is a difference between the total voltage of the bridge arm and the bus voltage, which leads to the generation of circulating current in the MMC.

\subsection{Pre-charging control strategy}

According to the characteristics of MMC topology, each MMC sub-module has an independent capacitor. Before starting MMC, the capacitor voltage of each sub-module is basically zero. If the system is operated directly without charging the sub-module capacitor, the system will generate particularly large inrush current, which will cause the MMC system to burn down. It is necessary to charge the capacitors of all sub-modules to make the 
capacitor voltage of each sub-module reach the rated working voltage.

According to the introduction in literature [8-12], the sub-module pre-charging can be divided into two stages: (1) the uncontrollable pre-charging stage: in the first stage, each sub-module is turned off, and the current is charged as the capacitor through the reverse parallel diode of each sub-module. (2) the controversiable precharging stage: in the second stage, the capacitor of the sub-module is further charged by controlling the switching states of the switching devices $V_{1}$ and $V_{2}$ in the sub-module. This paper aims at the uncontrollable charging stage, charging the sub-module through the DC side bus voltage.

Firstly, it is analyzed that in the uncontrollable charging stage, different values of current limiting resistors at the DC side will have different influences on the capacitance voltage value of the sub-module and the current value of the bus at the DC side. The corresponding relationship is shown in Figure 4 and Figure 5.

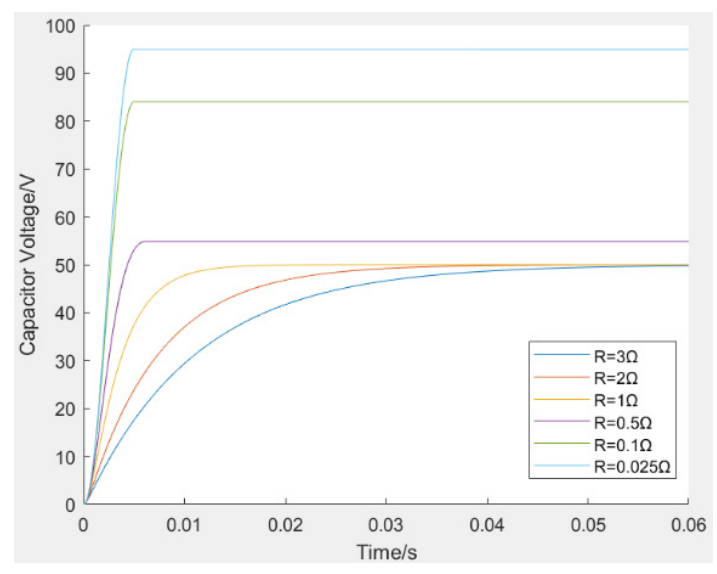

Fig. 4. Capacitor voltage of SM with different resistors

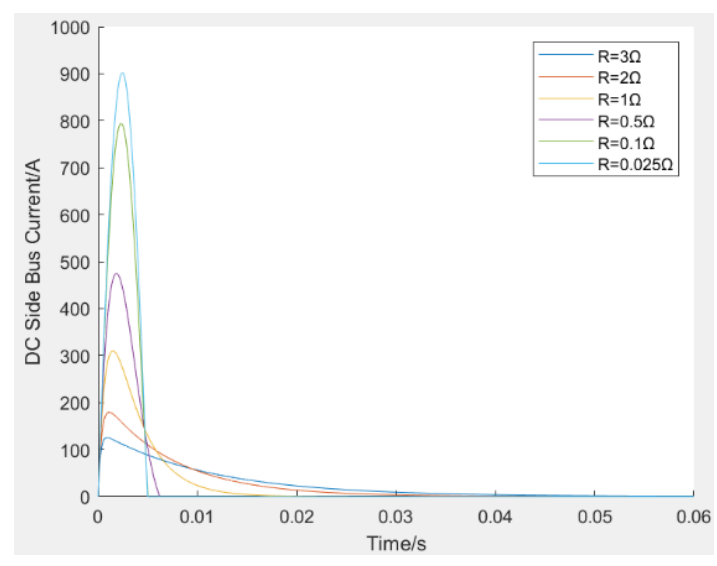

Fig. 5. Bus current under different resistances

As can be seen from Figure 4, as the resistance value of the DC side current limiting resistor gets smaller and smaller, at the end of the uncontrollable pre-charging stage, the capacitor voltage of the sub-module gets higher and higher and approaches its rated value constantly, and the time to reach the maximum voltage in the uncontrollable stage is also shorter and shorter. It can be seen from Figure 5 that the bus current on the DC side increases with the decrease of the current limiting resistance. According to the experimental results in Figure 4 and Figure 5, the resistance value of the current limiting resistor can be selected as small as possible within the range that the circuit device can withstand, so as to improve the capacitance voltage of the sub-module at the end of the uncontrollable stage of precharging and shorten the time to reach the voltage.

In the uncontrollable pre-charging stage, although reducing the current limiting resistor value can improve the voltage value of the capacitor voltage of the submodules in the uncontrollable stage, the current will increase with the decrease of the current limiting resistor. This paper proposes a control method in the uncontrollable pre-charging stage, which can suppress the current value of DC bus when the current limiting resistor resistance value is low. Under the condition that the current limiting resistor resistance value is fixed, a series of resistors are placed on the bus at the DC side, and the resistors are gradually bypassed after the circuit is turned on. This method will not occupy a lot of controller resources, the current limiting resistance can be controlled through the bypass operation of the system, the performance of the bypass switch is not too high requirements, improve the charging efficiency and control effect.

\section{Results \& Discussion}

A modular five-level inverter is built in Matlab/Simulink. Some parameters of the five-level inverter are set as follows:

Table 2. Simulation parameters of modular five-level inverter

\begin{tabular}{|c|c|}
\hline Parameter & Value \\
\hline DC bus voltage / V & 400 \\
\hline $\begin{array}{c}\text { Number of SM for a single } \\
\text { bridge arm }\end{array}$ & 4 \\
\hline SM capacitance / mF & 10 \\
\hline SM rated voltage / V & 100 \\
\hline Bridge arm inductance / mH & 2 \\
\hline
\end{tabular}

When the current limiting resistance $\mathrm{R}$ fixed, $\mathrm{m}$ resistors are connected in series at the $\mathrm{DC}$ side bus, and after the circuit is switched on, the $\mathrm{m}$ resistors are bypassed in turn. The bridge arm current can be greatly suppressed at the end of the uncontrollable stage at a little lower capacitance voltage than if this strategy is not used. The above method was experimentally verified by setting the current limiting resistor $\mathrm{R}=0.2 \Omega$, and connecting two additional resistors in series (i.e. $\mathrm{m}=2$ ). The resistance value of the first resistor was set as $0.8 \Omega$, and it was bypassed after the circuit was run $0.2 \mathrm{~ms}$. The 
second resistor is set to $0.2 \Omega$, and it was bypassed after the circuit was run $2 \mathrm{~ms}$. The results before and after this control strategy were shown in Figure 6 and Figure 7.

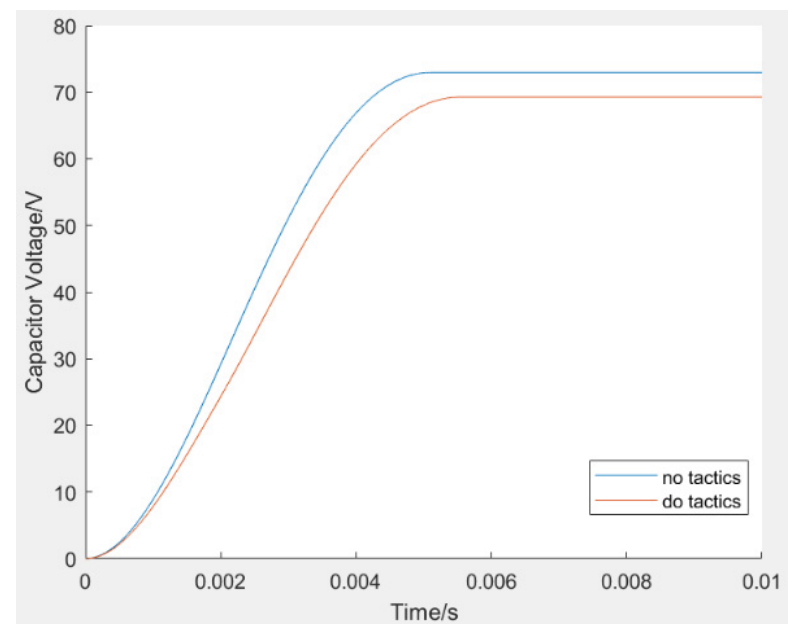

Fig. 6. Comparison of SM capacitance voltage

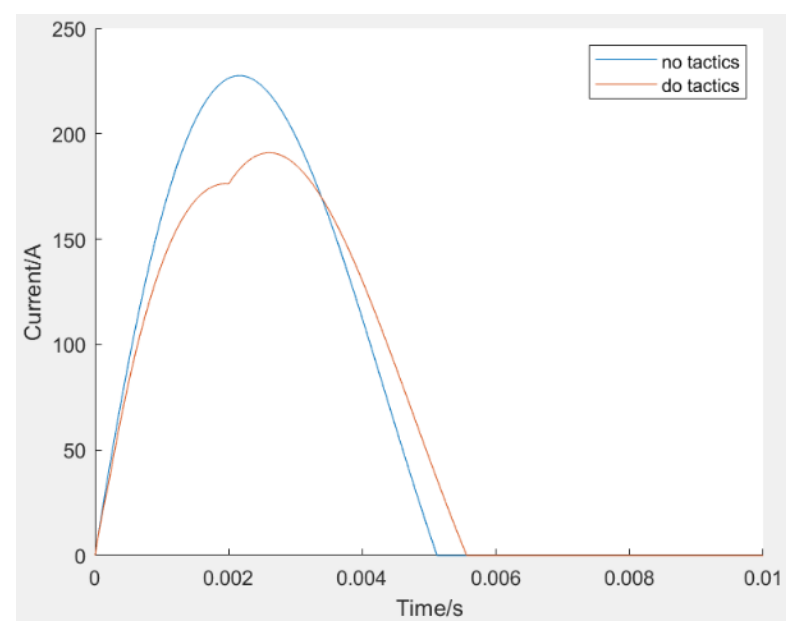

Fig. 7. Comparison of bridge arm current

As we can see from figure 6 and figure 7, after using this control strategy, uncontrollable stage at the end of the pre-charging module capacitance voltage less adopted this strategy reduces about $3 \mathrm{~V}$, but lower bridge arm current peak about $37 \mathrm{~A}$, validate the correctness of the above method, the control method to sacrifice a little voltage, greatly reduce the peak current control effect.

\section{Conclusions}

MMC due to its own characteristics, it has attracted wide attention in the field of direct current transmission. Before the MMC inverter works, it needs to be precharged. That is to say, the capacitor voltage of the submodule is charged near the rated value to make it better into the working state. Precharge can be divided into DC side precharge and AC side precharge according to charging mode, and can also be divided into controllable charging stage and uncontrollable charging stage according to whether the working state of the submodule can be controlled during the charging process. At present, experts and scholars have studied more AC side precharge, but there are few researches on DC-side pre-charging. In this paper, the uncontrollable charging stage of DC-side pre-charging is studied, and the influence of the value of current limiting resistance of the DC-side bus on the capacitance voltage of the submodule is analyzed in the uncontrollable charging stage of DC-side pre-charging. On this basis, the control idea of controlling the uncontrollable charging phase to reduce the current of the bridge arm is proposed, and the feasibility of the method is verified by simulation.

In the next step, the control idea proposed in this paper will be analyzed in detail, so as to realize a faster and more stable DC side pre-charging strategy.

\section{Acknowledgments}

Scientific Research Project of Shanghai Municipal Science and Technology Commission, "Research on Real-time Simulation and Grid-connection Test Platform for High-Power Wind Turbines and Certification Test Technology" (Project No.17DZ1201200).

\section{References}

1. Q.x. Zhu, G. Qi, Y.w. Wei, X.g. Wang. Industry and Mine Automation, 45(2019)

2. Y.1. Dong, J. Tian, X.m. Huang, X.b. Zhong, N.n. Wang, M.k. Liu. Automation of Electric Power Systems, 38(2014)

3. X.k. Wu, K. Wang, L. Wan, Z.d. Zheng, Y.d. Li. New Technology of Electrical Engineering and Energy, 12(2014)

4. J. Zhou, J.h. Su, X.y. Wang. High Voltage Apparatus, 50(2014)

5. Y. Jiang, Y. Huang, J.w. Hu, X. Wang, W.y. Luo, Z.m. Rao. Control Engineering of China, 22(2015)

6. P.w. Xie, S.b. Qian, F. Song. Ship Electric Technology, 37(2017)

7. P.w. Xie, Y.q. Zhao, C.h. Liu. Ship Electric Technology, 40(2020)

8. G.p. Guo, Hu Xuehao, J.1. Wen, G.1. Wu, T. Yao, J. Yang, X. You.Power Grid Technology, 38(2014)

9. Q.f. Zeng, F. Xiao, S. Ai, Q. Ren. Journal of Naval University of Engineering, 32(2020)

10. L.p. Wu, W.c. Ma, D. Yan, N. Zou. Power Electronics Technology, 54(2020)

11. Y.j. Liu, J.j Song, Y.j. Li, R.j. Zhu. Shandong Industrial Technology, 11(2017)

12. M. Kong, Y.f. Qiu, Z.y. He, W.g. He, J. Liu. Power System Technology, 35(2011) 